\title{
Performance of Comparative Cervical Tuberculin Test and Serological Methods with Culturing of Nasal Swab in Diagnosis of Bovine Tuberculosis in Cross Breed Cattle Baghdad Iraq: A Comparative Evaluation
}

\author{
Waffa A. Ahmed \\ Department of Microbiology, College of Veterinary Medicine, Baghdad University, Baghdad, Iraq \\ Email: waffaabduleah@yahoo.com
}

How to cite this paper: Ahmed, W.A. (2016) Performance of Comparative Cervical Tuberculin Test and Serological Methods with Culturing of Nasal Swab in Diagnosis of Bovine Tuberculosis in Cross Breed Cattle Baghdad Iraq: A Comparative Evaluation. Advances in Microbiology, 6, 867-878. http://dx.doi.org/10.4236/aim.2016.611082

Received: July 20, 2016

Accepted: September 16, 2016

Published: September 19, 2016

Copyright $\odot 2016$ by author and Scientific Research Publishing Inc. This work is licensed under the Creative Commons Attribution International License (CC BY 4.0).

http://creativecommons.org/licenses/by/4.0/

(c) (i) Open Access

\section{Abstract}

This study was designed to assess the diagnostic value of Rapid Antigen bovine TB antibody test kit (RAT) and any association with cervical comparative tuberculin test (CCTT), (iELISA) and nasal swabs culturing, among based detection of M. bovis infection. A herd of 21 animals aged $1-8$ years cross bread cattle of college of veterinary medicine. 19 (90.47\%) animals had good body condition scores, two bulls included, and $2(9.52 \%)$ cows were fair. Serum samples were collected, analyzed for anti-bovine TB antibody using RAT and iELISA. Also the herd was screened by CCTT. The tests were carried out twice, more than ten month interval, and twelve nasal swabs were taken within second survey. The first survey results revealed prevalence rate: $4(19.04 \%)$ animals considered positive results (one positive and 3 suspected results) for CCTT, while the prevalence rate according to RAT was 10 (47.61\%). The difference between the two prevalence rate was significant $(\mathrm{McNemar}$ chi-statistic $=$ $4.50, \mathrm{p}$-value $=0.03) \mathrm{Kappa}=0.21595 \%$ confidence interval: from -0.128 to 0.558 ; the strength of agreement is considered to be "fair". The study interprets: sensitivity $30 \%$; specificity $99 \%$. The second survey results revealed prevalence rate according to CCTT was 4 (36.36\%), while prevalence rate according to RAT was $5(45.45 \%)$. The difference between the two prevalence was not significant $(\mathrm{McNemar}$ chi-statistic $=$ 0.33 , p-value $=0.56$ ). Kappa $=0.44195 \%$ confidence interval: from -0.087 to 0.968 ; the strength of agreement is considered to be "moderate"; sensitivity: $60 \%$; specificity: $83 \%$. All serum samples and nasal swabs gave negative results for iELISA and culturing respectively. The study concluded that RAT was highly specific, easy, labor and time saving, suggesting its use as screening test in bovine tuberculosis, and 
CCTT could be used to confirm positive animals screened by RAT, while there was no association between RAT, CCTT with body condition scores, iELISA and nasal swabs culture results.

\section{Keywords}

BTB, Cervical Comparative Tuberculin Test, One Step Bovine TB Antibody Rapid Test, iELISA

\section{Introduction}

Bovine tuberculosis is not only an economic disease representing a barrier for free tread of livestock between countries but also a zoonotic disease with high prevalence in developing countries [1] [2].

Infection is often subclinical, when present clinical signs are not specifically distinctive of this disease, so it is usually diagnosed in the live animal on the basis of delayed hypersensitivity reaction [3]. It has been estimated that the tuberculin skin tests (TSTs) are the most important techniques for diagnosis of bovine TB in field [4] [5]. The single intradermal comparative cervical tuberculin test (SICCTT) involving the injection of BPPD intradermally and avian tuberculin at separate sites in the skin fold of the neck yields exceedingly specific results than the single skin injection test as codal fold tuberculin test which uses BPPD only [6] [7].

The tuberculin skin test is effective in early detection of pre-clinical cases of $M$. bovis in cattle. This allowed the rapid removal of infected animals, and thus limited transmission of the disease and had a good impact in the early eradication of the disease [8]. Although physical examination is needed for TST in the field, its simplicity and coast effectiveness have made it as reliable technique for disease surveillance in cattle over large area under investigation [8] [9].

Many factors which could influenced the results of TST performance are: environmental factors, host factors (status of immunity, genetics), nature of tuberculin used [4] [6] [7] [10], in addition to the ability of the test to detect positive cases relying on its sensitivity, specificity and distribution of the diseased cattle in herd under investigation [4].

Low responsiveness of TST test for both avian and mammalian tuberculosis has been reported in animals that exhibit advance disease stage, pre-parturient cows, or cows with confined infection of the udder and with localized infection, when lymphatic glands are inactive [11]. Many studies from different parts of the world have evaluated the diagnosis of bovine TB by TST test in comparison with other TB diagnostics marker including ESAT-6, Gamma Interferon test, florescence polarization, serological tests and post mortem detection of TB in various environmental conditions [4] [5] [8] [12]. However, negative TST result has been reported in animals which have encapsulated lesions confirmed caused by $M$. bovis in post mortem [11].

Bovine TB diagnosis based on serological methods has advanced. Since specific anti- 
gens have been used to enhance the specificity and sensitivity of these tests, an important antigen MPB70 which is $M$. bovis secreted protein has been used to detect $M$. bovis antibodies (IgM and IgG) [13]. Its sensitivity is $90 \%$ in comparison with BTB positive confirmed by bacterial isolation and $85 \%$ in comparison with PPD test. On other hand, the specificity was $98.6 \%$ against PPD test, and the test required no additional equipment and it was easy, labor and time saving [14].

Detection of BTB and its adverse impact on milk production of lactating cow had been studied by using Immunochromotographic assay (ICGA) as antigen Rapid Bovine $\mathrm{Tb} \mathrm{Ab}$ Test kit in Bangladesh for the first time [15].

While in Iraq, it was employed for first time in herd of buffaloed to examine the prevalence of BTB (31). For more accurate results, it was suggested that clinical information and other test such as Enzyme linked immune sorbent Assay (ELISA), should be applied to determine the true status of animals [14]. However, ELISA regarded as valuable complimentary tool to identify clinically undiagnosed cows that may be act as reservoirs and play a role in dissemination of the agent throughout herd [16]. But it has low value when it used in infected animals when they are in early stage of infection [17]. While rapid test kit alone may not be reliable for screening bovine TB and additional tests is required to validate its results [18]. However, tuberculin skin test and anti BTB antibody tests (lateral flow) when used in parallel presented an amended detection of BTB compared to the results of each test [19]. There are paucity in reports concerning prevalence of bovine tuberculosis in Iraq, especially in last three decades, this could attributed partly to the cost and unavailability of a reliable and less cumbersome diagnostic screening test and political will to diagnose and eradicate the disease in livestock need to be improved more importantly. This study was designated to assess the diagnostic value of RAT for sero-diagnosis of bovine tuberculosis and any correlation with (CCTT), iELISA and culturing of nasal swabs among based detection of $M$. bovis infections.

\section{Materials and Methods}

The current study was performed on a herd of cattle (cross bred) in the field of college of Veterinary Medicine College/Baghdad University in Abu Graib region Baghdad, Iraq, the herd selected due to pervious history of positive and suspected results of (CCTT), the herd included 21 apparently healthy animals aged $1-8$ years, 19 females and 2 males. The animals were clinically examined for debilitation, respiratory signs, ulcerating wound and animals health status were clinically evaluated and scored on a scale of ( $1-2$ good, 3 - 4 fair and 5 poor) prior to the tests [20]. The herd was screened by CCTT for bovine tuberculosis before more than ten months through a survey in a project by [21].

\subsection{Samples Collection}

- Blood samples

Twenty one samples were collected, advanced pregnant animals were excluded. 


\section{- Nasal swabs}

Twelve nasal swabs were taken from animals which were positive for RAT and positive or suspected for CCTT.

Three tests were conducted to evaluate the prevalence of bovine tuberculosis in the herd (CCTT, Rapid Antigen Ab bovine tuberculosis test and iELISA).

- Comparative cervical tuberculin test (CCTT)

CCTT was conducted on animals which were given positive results by the previous first RAT, and positive and suspected cases by the previous CCTT, a volume of $0.1 \mathrm{ml}$ each of Bovine and avian tuberculin was injected intradermal in the neck in the middle portion on the right side about $(10-15) \mathrm{cm}$ apart, after shaving the two areas. The test was read 72 hours after inoculation [22] [23].

\subsection{Analysis of Data}

The prevalence rate of bovine TB was counted by the formula:

$$
\text { Prevalence Rate }(\%)=\frac{\text { Number of positive animals }(+ \text { ve tuberculin tests })}{\text { Total number of animals tested }}
$$

Chi-square test was employed to evaluate the relation between the factors and the disease [24].

- Antigen rapid bovine tb ab test kit

The test was conducted on sera-samples at the same time of the first CCTT, and conducted again for positive and suspected cases for the first CCTT and the positive for the first Rapid Antigen Test, using specific antibodies for M. bovis, the plastic cover was striped and test kit was placed on, dry level surface for five minutes at room temperature, test serum ( 4 drops) was added slowly to the sample well by help of the specimen dropper. If the migration did not appeared after one minute, one extra drop of the serum was added to the sample well, then the test results were read within $20 \mathrm{~min}$. Presence of purple line in the result well of the kit is an indication of positive result [14].

- iELISA

The test was conducted by using a flat-bottomed wells polystyrene micro titer plates (Dynatech Labs., USA). Immulon 2 coated $(1 \mathrm{mg} /$ well) with bovine tuberculin purified protein derivative (PPD; Tecpar Brazil), blocked with $100 \mathrm{ml} /$ well of $0.5 \%$ casein. A pool of eight positive sera from culture-positive animals and pool of eight animals originated from farm clear of TB and were negative to skin reaction test were used as controls. Test samples and control sera were tested in duplicate and incubate with a 1:500 dilution of monoclonal anti-bovine-IgG conjugated with alkaline phosphatase (Sigma) and absorbencies are read at $405 \mathrm{~nm}$. Animals were considered as positive when optical density value (OD) was $>0.350$. The mean absorbency values were calculated from replicates wells for each serum, and samples showing coefficients values of greater than $15 \%$ were repeated. The difference in absorbance between wells containing antigen minus wells lacking antigens represents final OD.

- Culturing of nasal swabs 
The twelve nasal swabs were cultured on stone brink [25].

\subsection{Statistical Analysis}

Data analyses were conducted using SPSS for windows version $17.01 \%$. The agreement between CCTT and RAT was calculated using Kappa analysis [26].

\section{Results}

In Table 1, the herd under investigation showed no signs of debilitation, respiratory and ulcerated wounds, they were apparently healthy, 18 out of $21(85.71 \%)$ have good body conditions scores, but number of the apparently healthy animals gave positive results with CCTT and RAT.

The results presented in Table 1 show that out of 21 animal, 1 (4.761\%) animal gave positive result and $3(14.28 \%)$ animals gave suspected results, while the other animals gave negative results for the first CCTT. Those four and other six animals gave positive results for the first RAT, this mean that $10(87.61 \%)$ out of 21 serum samples gave positive results for the first RAT. while one out of 11 (9.09\%) gave positive result, and 3 (27.27\%) gave suspected result for the second CCTT, while 5 (45.45\%) gave positive results for the second RAT, the positive and 2 of suspected cases for the second CCTT were included in the five positive cases for the second RAT, that the male gave positive result for the first RAT was very fierce and we can performed $2^{\text {nd }}$ CCTT only, while we failed to obtain blood samples in spite of sedation, and the other two animals were in progressive pregnancy (Tables 2-5).

Table 6 showed that sensitivity of the first CCTT and RAT was $20 \%$ \& $30 \%$ respectively, while specificity was $100 \%$ and $99.00 \%$, no significant difference was appeared at $(\mathrm{P}<0.05)$ in the sensitivity and specificity of the first CCTT and RAT.

Table 7 showed the sensitivity of the second CCTT and RAT was $60 \%$ and $20.00 \%$ respectively, while specificity was $83.00 \%$ and $100 \%$ respectively. There was significant difference at $(\mathrm{P}<0.01)$ in the sensitivity and specificity. All the results of iELISA and culturing of nasal swabs were negative.

\section{Discussion}

The use of combination of cellular and by several authors is for diagnosis of BT but this uncommon procedure [27]-[29]. While the delayed-type hypersensitivity (DTH) reaction providing a clear indication of infection or exposure to etiological agent, formation of antibody appeared to be correlated to the range of bacterial multiplication and antigenic cargo in the diseased individual [9]. The results showed number of apparently healthy animals gave positive or suspected results for bovine TB, this results was in agreement with Mellroy et al. [30] who reported that most M. bovis infected cattle appear normal, but they harbor and/or they may disseminate the bacterium for years while did not express signs of illness. This has posed a significant public health concern, especially in infected lactating cow which shedding the bacterium through milk which can be consumed by humans as unpasteurized raw milk this has been practiced in the 
study region.

Bovine tuberculosis prevalence in the herd was $4.76 \%$ and $36.36 \%$ by the first and the second CCTT respectively which was higher than prevalence $0.32 \%$ and $1.38 \%$ in cattle and buffaloes respectively, which was reported within a survey on bovine tuberculosis by CCTT in the middle and south of Iraq [31], also it was higher than $3.8 \%$ in a herd of buffaloes reported by Ahmed et al. [32]. And higher than 2.8\% in a herd of cattle reported by [18], while it was lower than $11.4 \%$, reported by [33].

Table 1. Results of CCTT, RAT and body condition scores, in cross bred cattle herd, used for detection of bovine $\mathrm{TB}$

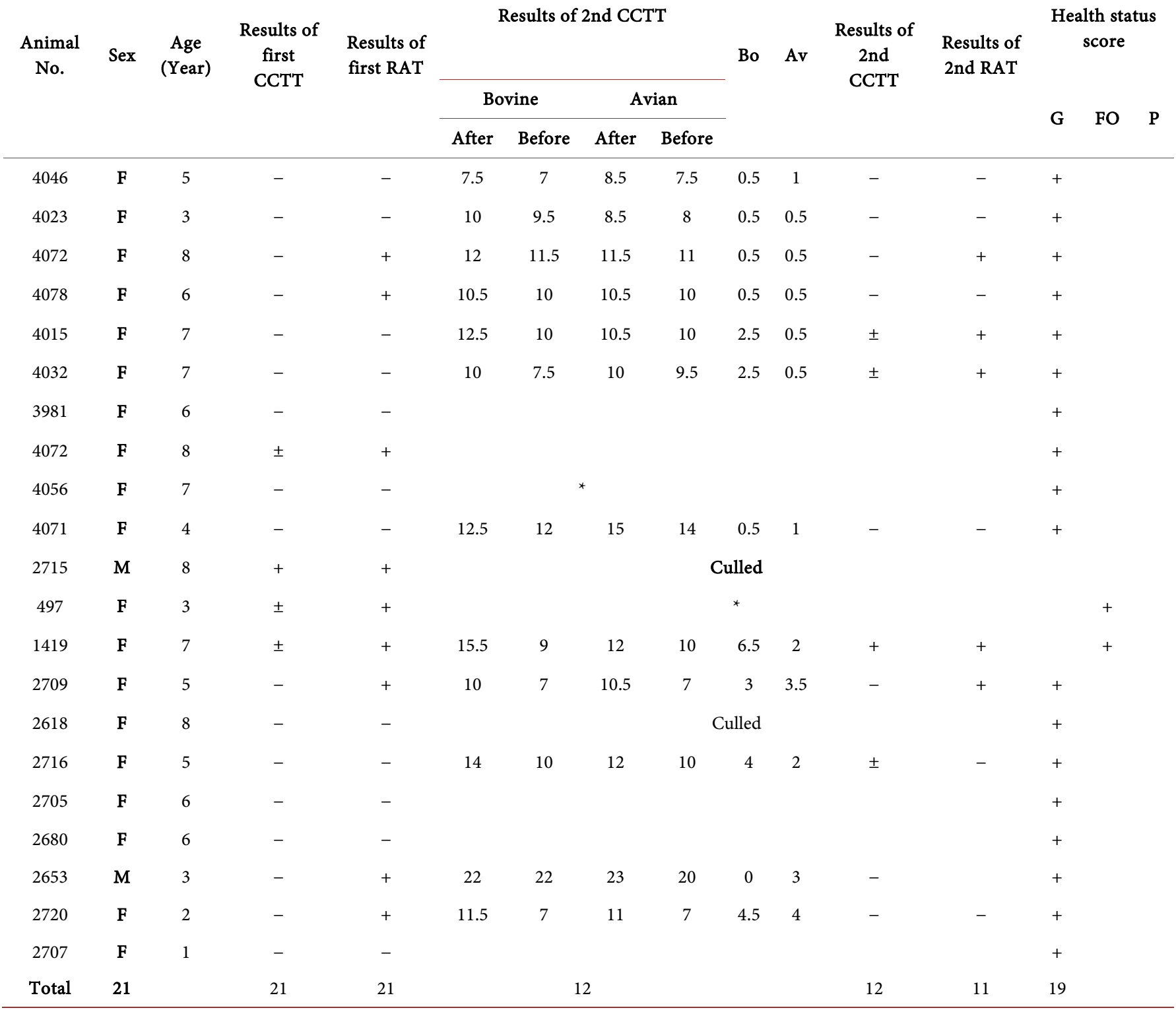

CCTT: Cervical Comparative Tuberculin Test; RAT: Rapid Antigen bovine TB antibody Test; F: female; M: male; G: good; FO: fair; P: poor; ${ }^{\star}$ Advance pregnancy: excluded in the second survey. 
Table 2. Show association between first CCTT and RAT when the suspected cases in CCTT considered as uninfected.

\begin{tabular}{cccc}
\hline & & First RAT & Total \\
\hline First CCTT & + & - & \\
+ & 1 & 0 & 1 \\
- & 9 & 11 & 20 \\
Total & 10 & 11 & 21 \\
\hline
\end{tabular}

The prevalence rate according CCTT $=1 / 21=4.76 \%$; The prevalence rate according RAT $=10 / 21=47.61 \%$; The difference between the two prevalence was significant (McNemar chi- statistic $=9.00$, P-value $<0.01)$; Kappa $=0.104$ $95 \%$ confidence interval: From -0.092 to 0.301 the strength of agreement is Considered to be "poor". Sensitivity $=$ 10 , Specificity $=100$.

Table 3. Show association between first CCTT and RAT when the suspected cases in CCTT considered as infected.

\begin{tabular}{cccc}
\hline & & First RAT & Total \\
\hline First CCTT & + & - & \\
+ & 3 & 1 & 4 \\
- & 7 & 10 & 17 \\
Total & 10 & 11 & 21 \\
\hline
\end{tabular}

The prevalence rate according CCTT $=4 / 21=19.04 \%$; The prevalence rate according RAT $=10 / 21=47.61 \%$; The difference between the two prevalence rate was significant $(\mathrm{McNemar}$ chi-statistic $=4.50$, $\mathrm{p}$-value $=0.03)$; Kappa $=$ $0.21595 \%$ confidence interval: From -0.128 to 0.558 the strength of agreement is considered to be "fair"; Sensitivity $=30 \%$, Specificity $=99 \%$.

Table 4. Show association between second CCTT and RAT when the suspected cases in CCTT considered as infected.

\begin{tabular}{|c|c|c|c|}
\hline \multirow[b]{2}{*}{ Second CCTT } & \multicolumn{2}{|c|}{ Second RAT } & \multirow[t]{2}{*}{ Total } \\
\hline & + & - & \\
\hline+ & 3 & 1 & 4 \\
\hline- & 2 & 5 & 7 \\
\hline Total & 5 & 6 & 11 \\
\hline
\end{tabular}

The prevalence rate according CCTT $=4 / 11=36.36 \%$; The prevalence rate according RAT $=5 / 11=45.45 \%$; The difference between the two prevalence was not significant $(\mathrm{McNemar}$ chi-statistic $=0.33$, p-value $=0.56)$; Kappa $=$ $0.44195 \%$ confidence interval: From -0.087 to 0.968 the strength of agreement is considered to be "moderate"; Sensitivity $=60 \%$, Specificity $=83 \%$.

Table 5. Show association between second CCTT and RAT when the suspected cases in CCTT considered as uninfected.

\begin{tabular}{cccc}
\hline & & Second RAT & Total \\
\hline Second CCTT & + & - & \\
+ & 1 & 0 & 1 \\
- & 4 & 6 & 10 \\
Total & 5 & 6 & 11
\end{tabular}

The prevalence according CCTT $=1 / 11=9.09 \%$; The prevalence according RAT $=5 / 11=45.45 \%$; The difference between the two prevalence was significant $(\mathrm{McNemar}$ chi-statistic $=4.00$, $\mathrm{p}$-value $=0.04)$; Kappa $=0.21495 \%$ confidence interval: From -0.166 to 0.594 the strength of agreement is considered to be "fair"; Sensitivity $=20 \%$, Specificity $=100 \%$. 
Table 6. Show comparison between sensitivity and specificity of the first CCTT and RAT .

\begin{tabular}{ccc}
\hline Diagnostic test & Sensitivity & Specificity \\
\hline First CCTT & $20.00 \%$ & $100.0 \%$ \\
First RAT & $30.00 \%$ & $99.00 \%$ \\
P-value & $0.0477^{\star}$ & $0.561 \mathrm{NS}$ \\
\hline
\end{tabular}

$*(\mathrm{P}<0.05)$.

Table 7. Show comparison between sensitivity and specificity of second CCTT and RAT.

\begin{tabular}{ccc}
\hline Diagnostic test & Sensitivity & Specificity \\
\hline Second CCTT & $60.00 \%$ & $83.00 \%$ \\
Second RAT & $20.00 \%$ & $100.0 \%$ \\
P-value & $0.0031^{* *}$ & $0.0149^{* *}$ \\
\hline
\end{tabular}

${ }^{* *}(\mathrm{P}<0.01)$, NS: Non-significant.

The tuberculin skin test results may be false positive because several factors including status of immunity of the animal and nature of tuberculin used [1] [10]. Also may be attributed to sensitization of Mycobacteria spp. other than M. bovis [25]. The small size of the herd and good health status scores may explain the low disease prevalence in the herd which may have not been in contact elsewhere with any infected herds [34] pointed out that increased in herd size, increases the risk of positive reactors to the tuberculin test, this may be due to increasing herd size, so the probability of introduction of infected animals to the negative herd become higher.

Bovine tuberculosis prevalence in the herd by the first and the second RAT was 10 (47.61\%) and 5 (45.45\%) respectively, these results were lower than (10/16) 62\% reacted positively for M. bovis antibodies with IQRT while no postmortem Tb gross lesion was observed from the six randomly selected cows out of ten IQRT positive cows culled and examined [15].

But the results disagreed with [18] who pointed out that all animals showed no visible reaction to the quicking bovine tuberculosis antibody rapid test but seven animals reacted positively to skin test in Jalingo, Nigera.

The unique feature of bovine tuberculosis represented by the difficulty of using an accurate rapid and simple diagnostic test alone, most of the tests have a low sensitivity, led to requirement of adopting more than one test for detection, hence the results of current study confirmed that the efficiency of detecting BT differs by CCTT \& RAT. In the first survey, prevalence rate detected by RAT was much higher than that of CCTT, but in the second survey, prevalence rate detected by RAT was similar with that of CCTT, this may be due to the small number of serum samples, which were taken from only [11] animal, included in the second survey.

The most interesting result of this study is the co efficient of kappa between the two tests, which revealed the strength of agreement which was ranged for poor fair and moderate value, the results could include the suspect case with CCTT when subjected to RAT all of them showed positive results in the first survey, while only two of three of 
the suspected case with CCTT showed positive results in the second survey.

The results of current study clarified that the RAT was higher in sensitivity than CCTT in the first survey, while CCTT was higher in sensitivity than RAT of the second survey; these results were agreed with [14] who reported that rapid ICA was found to be more sensitive than SCCTT while SCCTT was found to be more specific than rapid ICA, as rapid ICA can capture and detect M. bovis antibodies (IgM and IgG), due to the recombinant MPB70 antigen which act as capturer and detector material, this antigen is present predominantly in M. bovis and to very limited degree in M. tuberculosis and other Mycobacteria spp., so the kit test doesn't have cross-reaction with other SPP. Also the result revealed low value for sensitivity rate compared with results obtained by clinical evaluated study by [14], which clarified that the sensitivity of RAT was $85 \%$ vs. PPD test and $90 \%$ vs. culture of $M$. bovis. Also the current study revealed that the rate of specificity for the two tests in the first survey was similar, while the rate of specificity of RAT was (100\%) which was significantly higher than CCTT in the second survey, these results agreed with [14] who reported specificity rate for RAT, 98.6\% vs. PPD test. While these results disagreed with [18] who pointed out that rapid test kit alone may not be reliable for screening bovine TB and additional tests is required to validate it's results.

The frequently use of culture method remains the gold standard for confirmation of TB. However, the time required for the isolation and biochemical analysis for identification of Mycobacteria, can take up eight weeks [35], but the negative nasal swabs culturing in the current study, was in agreement with the negative nasal swabs culture obtained by [21] and [36] who clarified that the negative results cannot be counted evidence of the absence of infection, this may be interpreted by intermittent appearance of $M$. bovis in some nasal swabs or may be (a lag period) in the shedding of the bacteria after infection. This may also indicate a phenotype change in the microbe and/ or obtain overlapping with the host, probably due to environment stress factor and physiology of the host of various kinds and that play a role as agents involved in the time shedding of germ [36].

The negative results for the positive and suspected infected animals for RAT and CCTT, by iELISA may be attributed to the early stage of infection, which was inconsistent with [17] who clarified that ELISA has low value when it is used in animals which were in early stage of infection. Its sensitivity is limited mostly because of the late and irregular development of the humeral immune response in cattle during the course of the disease, and also specificity is poor in cattle when complex antigens such as tuberculin or M. bovis culture filtrates are used [37]. All the data recovered from the tests used in this study confirmed the sophisticated nature of immune response to Bovine tuberculosis and there is no precise parameter for diagnosis of clinical or subclinical carrier cases which play a major role of BT bacilli spreading to other healthy animals and humans.

\section{Conclusions}

The study concluded that RAT revealed high specificity, similar to CCTT, and occasio- 
nally higher or lower sensitivity than CCTT. Also it is easy, labor and time saving, suggesting its use as screening test in bovine tuberculosis, and the CCTT could be used to confirm positive animals screened by RAT. RAT could be useful in diagnosis of early infection of cattle with $M$. bovis, especially during test and slaughter program as infected cattle may be detected for culling early before losing their marketing value to condemned carcass during meat inspection.

We advise Iraqi government should assist livestock farmers in screening their herds for tuberculosis by using both RAT and CCTT, while insuring that infected animals are culled for slaughter and compensated.

\section{Acknowledgements}

I am extremely thankful to my colleagues of the research zoonotic unite especially Assistant Professor Nagham. M. Ayal, Assistant lecturers Ammer. A. S. Hatemm and Shaimaa A. Majeed, also for Wathique. A (veterinarian administrator) of the field of the college of veterinary medicine and to Elham Butris (head of the central laboratory of Kurdistan), for kindly supplying all the necessary facilities which were essential in iELISA and without them, this work would not been accomplished.

\section{References}

[1] Amanfu, W. (2006) The Situation of Tuberculosis and Tuberculosis Controlling Animals of Economical Interest. Tuberculosis, 86, 330-335. http://dx.doi.org/10.1016/j.tube.2006.01.007

[2] Feliciano, M.S., Beth, H., Camila, A.D., Cecilia, R.T., Tod, S., Genoveva, A.O., Alberto, M.L., Martina, P.S. and Janet, B.P. (2008) Molecular Epidemiology of Mycobacterium bovis. Usefulness in International Trade. Preventive Veterinary Medicine, 87, 261-271. http://dx.doi.org/10.1016/j.prevetmed.2008.04.004

[3] Wood, P.R. and Rothel, J.S. (1994) In Vitro Immunodiagnostic Assays for Bovine Tuberculosis. Veterinary Microbiology, 40, 125-135. http://dx.doi.org/10.1016/0378-1135(94)90051-5

[4] Domenech, R., Goodchild, T., Vordermeier, M. and Clifton-Hadley, R. (2006) Ante Mortem diagnosis of Bovine Tuberculosis: The Significance of Unconfirmed Test Reactors. Government Veterinary Journal, 16, 65-71.

[5] Rua-Domenech, R., Goodchild, A.T., Vordermeier, H.M., Hewinson, R.G., Christiansen, K.H. and Clifton-Hadley, R.S. (2006) Ante Mortem Diagnosis of Tuberculosis in Cattle: A Review of the Tuberculin Tests, Y-Interferon Assay and Other Ancillary Diagnostic Techniques. Research in Veterinary Science, 81, 190-210.

http://dx.doi.org/10.1016/j.rvsc.2005.11.005

[6] Monaghan, M.L., Doherty, M.L., Collins, J.D., Kazda, J.F. and Quinn, P.J. (1994) The Tuberculin Test. Veterinary Microbiology, 40, 111-124. http://dx.doi.org/10.1016/0378-1135(94)90050-7

[7] Francis, J., Choi, C.L. and Frost, A.J. (1973) The Diagnosis of Tuberculosis in Cattle with Special Reference to Bovine PPD Tuberculin. Australian Veterinary Journal, 49, 246-251. http://dx.doi.org/10.1111/j.1751-0813.1973.tb05211.x

[8] Buddle, B.M., Livingstone, P.G. and Del Lisle, G.W. (2009) Advances in Ante-Mortem Diagnosis of Tuberculosis in Cattle. New Zealand Veterinary Journal, 57, 173-180. http://dx.doi.org/10.1080/00480169.2009.36899 
[9] Monarchan, M.L. (1994) The Tuberculin Test. Veterinary Microbiology, 40, 111-124. http://dx.doi.org/10.1016/0378-1135(94)90050-7

[10] Ameni, G., Hewinson, G., Aseffa, A.A., Young, D. and Vorder-Meier, M. (2008) Appraisal of Interpretation Criteria for the Comparative Intradermal Tuberculin Test for Diagnosis of Tuberculosis in Cattle in Central Ethiopia. Clinical and Vaccine Immunology, 15, 12721276. http://dx.doi.org/10.1128/CVI.00114-08

[11] Good, M. and Duignan, A. (2011) Perspectives on the History of Bovine TB and the Role of Tuberculin in Bovine TB Eradication. Veterinary Medicine International, 2011, Article ID: 410470.

[12] Ngandolo, B.N.R., Muller, B. and Diguimbaye-Djaibe, C.J. (2009) Comparative Assessment of Fluorescence Polarization and Tuberculin Skin Testing for the Diagnosis of Bovine Tuberculosis in Chadian Cattle. Preventive Veterinary Medicine, 89, 81-89.

[13] Garnier, T., Eiglmeier, K., Cadmus, J.C, Medina, M. and Mansoor, H. (2003). The Complete Genome Sequence of Mycobacterium bovis. Proceedings of the National Academy of Sciences of the United States of America, 100, 7877-7882. http://dx.doi.org/10.1073/pnas.1130426100

[14] Quicking Biotect Company Ltd. (2012) Quicking Bovine Tuberculosis Antibody Rapid Test. http://www.e-to-china.com/

[15] Danabirni, S., Sackey, A.K.B., Kudi, A.C., Okaiyeto, S.O. and Pewan, S.B. (2009) Comparison of One-Step Antigen Rapid Bovine Tuberculosis Antibodies Test Sensitivity to Postmortem Gross Lesions in Diagnosis Bovine Tuberculosis in a Diary Sciences Herd in Kaduna State. Research Journal of Diary Sciences, 3, 32-34.

[16] Lilenbaum, W. and Fonseca, L. (2006) The Use of ELIZA as a Complementary Tool for Bovine Tuberculosis Control in Brazil. Department of Microbiology, Universidad Federal Fluminense, Niteroi.

[17] Thoen, C.O. and Ebel, E.D. (2006) Mycobacterium Bovis Infection in Animals and Humans. Chapter 6: Diagnostic Test for Bovine Tuberculosis, 2nd Edition, Blackwell Publishing, Ames, 49-53. http://dx.doi.org/10.1002/9780470344538

[18] Danbirni, S., Okaiyeto, S.O., Bature, C. and Moris, A. (2013) Field Determination of Tuberculosis Prevalence in a Herd of Cattle Using Tuberculin and Quicking ${ }^{\oplus}$ Bovine Tuberculosis Antibody Rapid Tests in Jalingo, Nigeria. Journal of Veterinary Advances, 3, 20-23.

[19] Awah-Ndukum, J., Kudi, A.C., Bah, G.S., Bradley, G., Tebug, S.F., Dickmu, P.L., Njakoi, H.N. and Agharih, W.N. (2012) Bovine Tuberculosis in Cattle in the Highlands of Cameroon: Seroprevlence Estimate and Rates of Tuberculin Skin Test Reactor at Modified Cut-Offs. Veterinary Medicine International, 2012, Article ID: 798502. http://dx.doi.org/10.1155/2012/798502

[20] Awah-Ndukum, J., Kudi, A.C., Bradley, G. and Bah, G.S. (2011) Assessment of Cut-Off Points during Tuberculin Skin Test for the Diagnosis of Bovine Tuberculosis in Cameroonian Cattle. Proceedings of the Annual Conference for British Society of Animal Science / Animal Science Forum and the Association of Veterinary Teaching and Research Work, Nottingham, 4-5 April 2011.

[21] AL-Ali, M.A.M. (2013) Diagnostic Study on Tuberculosis in Human and Cattle in Baghdad Province. A Thesis for the Degree of Master of Sciences in Veterinary Medicine, Zoonotic Diseases of the College of Veterinary Medicine, University of Baghdad, Baghdad.

[22] Chauhan, R.S. (1995) Vet. Clinical and Laboratory Diagnosis Japee Brothers (Pvt) Ltd., India, 230-233.

[23] Grooms, D. and Molesworth, J. (2000) The Comparative Cervical Tuberculin Test. Exten- 
sion Bulletin, E-2731, New September.

[24] Khan, I.A., Khan, A., Mubarak, A. and Ali, S. (2008) Factors Affecting Prevalence of Bovine Tuberculosis in Nili Ravi Buffaloes. Pakistan Veterinary Journal, 28, 155-158.

[25] Quinn, P.J., Markey, B.K., Carter, M.E., Donnally, W.J.C. and Leonard, F.C. (2006) Veterinary Mirobilogy \& Microbial Disease. TJ International LTD Pad Tow, Cornwall.

[26] SPSS (Statistical Package for the Social Sciences) (2016) Vol. 23, Stanford, VSA.

[27] Placket, P. (1989) An ELISA for the Detection of Anergic Tuberculous Cattle. Australian Veterinary Journal, 66, 15-19. http://dx.doi.org/10.1111/j.1751-0813.1989.tb09706.x

[28] Lepper, A.W., Pearson, C.W. and Corner, L.A. (1977) Serological Responses in Experimental Bovine Tuberculosis. Australian Veterinary Journal, 53, 301-305. http://dx.doi.org/10.1111/j.1751-0813.1977.tb00236.x

[29] Lightbody, K.A. (1998) Mycobacterial Antigen Specific Antibody Responses in Bovine Tuberculosis: An ELISA with Potential to Confirm Disease Status. The Veterinary Record, 142, 295-300. http://dx.doi.org/10.1136/vr.142.12.295

[30] Mellroy, S.G., Neill, S.D. and McCracken, R.M. (1986) Lesions and Mycobacterium Bovis Excreting Respiratory Tracts of Tuberculin Reacting. Veterinary Record, 118, 718-721. http://dx.doi.org/10.1136/vr.118.26.718

[31] Real Situation of Veterinary Office (2015) Report on Results of Tuberculosis Survey in Cattle and Buffaloes in the Middle and South of Iraq.

[32] Ahmed, W.A., Abdul Ameer, A.H., Al-Juburi, N.M., Butris, E. and Salih, I.K. (2015) Detection of Bovine Tuberculosis by Comparative Cervical Tuberculin Tests, Antigen Rapid Test and IELISA in a Buffaloe (Babalusbabalis) Herd in Baghdad. 5th Annual Conference for Development of Buffaloes, 23 April 2015, 70-79.

[33] Danbirni, S., Okaiyeto, S.O., Joshua, I.A., Sackey, K.B., Anthony, K.C., and Abdulkadir, I.A. (2012) Prevalence of Tuberculosis in a Herd of Cattle of a Tuberculous Herdman Following Trace Back Information from a Hospital in Taraba State, Nigeria. Journal of Animal Production Advances, 2, 325-328.

[34] Ameni, G. and Erhikun, A. (2007) Bovine Tuberculosis in Small-Scale Dairy Farms in Adama Town, Central Ethiopia, and Farmers' Awareness of the Disease. Revue Scientifique et Techniques de Poffice International des Epizooties, 24, 711-719. http://dx.doi.org/10.20506/rst.26.3.1778

[35] Maurico, M.N., Etoy, F.A. and Berry, W. (2003) Extraction of Mycobacterium Tuberculosis DNA: A Question of Containment. Journal of Clinical Microbiology, 43, 2996-2997.

[36] Al-Sadii, B.Q. (1999) Preparation of Tuberculins from Local Isolate of Mycobacterium bovis with an Immunocomparative Study with Nocardia asteroides. PhD Thesis, Veterinary Medicine, Baghdad University, Baghdad.

[37] Griffin, J.F.T., Hesketh, J.B., Mackintosh, C.G., Shi, Y.E. and Buchan, G.S. (1993) BCG Vaccination in Deer: Distinctions between Delayed Type Hypersensitivity and Laboratory Parameters of Immunity. Immunology and Cell Biology, 71, 559-570.

http://dx.doi.org/10.1038/icb.1993.62 
Submit or recommend next manuscript to SCIRP and we will provide best service for you:

Accepting pre-submission inquiries through Email, Facebook, LinkedIn, Twitter, etc. A wide selection of journals (inclusive of 9 subjects, more than 200 journals)

Providing 24-hour high-quality service

User-friendly online submission system

Fair and swift peer-review system

Efficient typesetting and proofreading procedure

Display of the result of downloads and visits, as well as the number of cited articles

Maximum dissemination of your research work

Submit your manuscript at: http://papersubmission.scirp.org/ 